\title{
Política sobre o uso da tecnologia da informação para a melhoria dos cuidados da saúde rural
}

\author{
Aprovado por Wonca World Council Meeting
}

12 de junho de 1998

Wonca Rural Information Technology Exchange (WRITE)

Esta Política sobre o uso da Tecnologia da Informação para a Melhoria dos Cuidados da Saúde Rural da Wonca foi preparada pela Wonca Rural Information Technology Exchange (WRITE), na Subcomissão do Grupo de Trabalho sobre Formação da Prática Rural da Wonca.

\section{Sumário Executivo}

A Tecnologia da Informação e Comunicação possui um potencial considerável para que haja impactos positivos ou negativos sobre o acesso à prestação dos serviços de saúde rural. Esta declaração política proporciona aos participantes, financiadores e planejadores dos serviços de saúde rural orientaçôes para a introdução e uso da Tecnologia da Informação e Comunicação para as comunidades rurais.

As comunidades rurais foram as primeiras a adotar essa tecnologia, sendo que essas tecnologias podem reduzir seu isolamento e desvantagem no acesso aos serviços. Com baixo nível, e custo relativamente baixo, as tecnologias têm o potencial de oferecer acesso a recursos de informação úteis para muitas comunidades rurais, especialmente os serviços baseados na Internet. Se uma comunidade rural tem acesso a serviços básicos de telefonia e a um computador, então essa comunidade tem acesso a e-mail como em um serviço de Internet básico. Neste nível básico, há muitas oportunidades para usar o e-mail para reduzir o isolamento profissional dos trabalhadores da saúde rural.

Planejadores e designers da Tecnologia da Informação e Comunicação devem reconhecer o impacto econômico de tais serviços em uma comunidade rural ao especificar componentes e critérios de desempenho. Muitas comunidades rurais não são capazes de manter os serviços de Tecnologia da Informação e Comunicação, sem significativos subsídios do centro de financiadores. Os financiadores dos Serviços de Tecnologia da Informação e Comunicação Rurais também devem alocar níveis suficientes de financiamento recorrente, para que os trabalhadores de saúde rural tenham acesso ao suporte técnico e à formação contínua e para substituição de capital de atualização e depreciação dos custos nos equipamentos da tecnologia da informação. 
Fatores críticos no planejamento e implementação que irão determinar o sucesso e sustentabilidade dos serviços de Tecnologia da Informação e Comunicação incluem:

- as necessidades apropriadas e levantamento ativo das comunidades rurais para o planejamento dos Serviços de Tecnologia da Informação e Comunicação;

- uma parceria envolvendo os financiadores e planejadores com as comunidades rurais que reconheça, respeite e responda às opinióes e necessidades dos trabalhadores de saúde local, em todas as fases do planejamento e implementação destes serviços;

- níveis suficientes de financiamento para a saúde rural, para apoiar um compromisso político para a saúde rural, incluindo a Tecnologia da Informação e Comunicação;

- o potencial de parcerias locais, nacionais, regionais e mundiais que podem aumentar o nível dos serviços prestados e/ou distribuir o custo, ao fornecer infraestrutura de tecnologia da informação e comunicaçóes; e

- consideração sobre se existe alguma barreira regulatória para a Tecnologia da Informação e Comunicação (especialmente para o reembolso no fornecimento dos cuidados de telessaúde) e iniciativas para garantir que estas serão tratadas antes da introdução de tais serviços.

A adoção de serviços de Tecnologia da Informação e da Comunicação não deve ter um impacto adverso sobre o acesso aos serviços de saúde por parte das comunidades rurais. O planejamento dos serviços de Tecnologia da Informação e Comunicaçáo nunca deve ser destinado a substituir os serviços de saúde locais na assistência prestada por profissionais de saúde rurais para a comunidade em nível local.

Qualquer prestação de serviços baseada em telessaúde deve reconhecer a referência existente e os padrôes de acesso entre as comunidades rurais e os prestadores dos cuidados de saúde secundários/terciários. A instalaçáo das tecnologias da informação e comunicação para apoiar o telessaúde também oferece aos trabalhadores de saúde rural, a oportunidade de acesso a uma gama de serviços de educação continuada a distância.

Há um pequeno corpo de evidências até o momento que dá suporte à eficácia da tecnologia da informação e comunicação, mas os futuros programas devem incorporar a coleta de dados e a análise do processo, o impacto e os resultados da Tecnologia da Informação e Comunicação, como um componente central do processo de planejamento. A avaliação deve ser parte integrante de quaisquer Serviços de Tecnologia da Informação e Comunicação. Os critérios de avaliação devem permanecer sensíveis às questóes locais das comunidades rurais.

\section{Recomendações para a Tecnologia da Informação e Comunicação Rural}

\section{Parceria com a Comunidade}

1. O planejamento dos programas de tecnologia da informação e de comunicaçóes deve reconhecer e responder especificamente às necessidades e expectativas locais da comunidade rural e de seus trabalhadores de saúde. A participação ativa das comunidades rurais e de seus profissionais de saúde deve ser um componente integral em toda a fase do processo de planejamento e implementação das tecnologias da informação e comunicação.

\section{Especificaçáo e Design do Sistema}

2. Uma parte integral para estabelecer os programas de tecnologia da informação e comunicaçáo é o de assegurar que o sistema a ser implementado adere às recomendaçóes de estrutura de dados e às normas e protocolos de comunicação para arcar com o cumprimento ideal dos sistemas regional, nacional e internacional.

\section{Segurança e Confidencialidade}

3. A especificação de protocolos de segurança de tecnologia da informação e comunicação deve aderir aos padrōes internacionalmente reconhecidos. A implementação destas normas não deve impactar negativamente os trabalhadores rurais da saúde. 


\section{Questóes locais}

4. As comunidades rurais devem ter acesso aos serviços de telecomunicaçôes e largura de banda capaz de fornecer pelo menos um nível mínimo aos serviços de tecnologia da informação e comunicação para as necessidades identificadas.

5. As comunidades rurais não devem ser penalizadas por quaisquer alteraçôes no ambiente econômico local, criadas quando os sistemas de comunicação são instalados para apoiar as tecnologias da informação e comunicação.

\section{Implementaçáo - Questóes Local, Regional e Mundial}

6. As comunidades rurais devem procurar trabalhar com outras partes interessadas em empreendimentos cooperativos, para melhorar a infraestrutura das comunicaçōes locais.

7. Todos os países, em particular os países em desenvolvimento, devem ser encorajados a procurar ativamente relaçôes de parceria regional e mundial com Universidades, indústrias e fundaçôes em outros países, para desenvolver e apoiar a disseminação das tecnologias da informação e comunicação nas áreas rurais.

\section{Implementação - Questóes Regulatórias}

8. As questóes políticas e decisôes relativas à tecnologia da informação e comunicação não devem afetar adversamente a distribuição local dos cuidados de saúde nas comunidades rurais.

9. As questóes de regulamentação e barreiras que possam ter impacto sobre a distribuição interestadual ou internacional dos serviços de tecnologia da informação e comunicação devem ser claramente identificadas e tratadas em nível nacional.

\section{Implementação - Questóes de Reembolso}

10. O planejamento e implementação de serviços de telessaúde devem abordar a questão do reembolso das partes envolvidas no processo de teleconsulta.

\section{Implementação - Financiamento em Curso}

11. O financiamento para tecnologia da informação e comunicação deve incluir alocações para fornecer níveis adequados de despesas correntes para apoio técnico, manutenção e modernização do equipamento.

\section{Implementaçáo - Formaçáo de Pessoal}

12. Uma promoção abrangente e um programa de treinamento no uso e aplicação das tecnologias da informação e comunicação para a saúde, incluindo a divulgação de experiências de projetos existentes, são componentes imperativos de qualquer projeto de tecnologia da informação e comunicação. Tais programas devem reconhecer as diversas e variadas necessidades dos trabalhadores de saúde rural e dos clientes e comunidades para os quais prestam serviços.

13. Todos os projetos que envolvam a aplicação de tecnologia da informação e comunicação para a saúde, financiados pelas autoridades de saúde nacionais, devem ser obrigados a ter uma formação no manejo da tecnologia integrada dentro do projeto e conhecer as questóes culturais e organizacionais, que sáo partes integrantes para o sucesso da tecnologia da informação e comunicação implementada.

\section{Implementação - Avaliação}

14. O desenvolvimento e medição do processo, impacto e critério de avaliação dos resultados dos serviços de tecnologia da informação e comunicação devem ser componentes fundamentais de qualquer programa de tecnologia da informação e comunicaçáo e devem ser baseados em fatores que são relevantes e importantes para as comunidades rurais envolvidas.

15. As autoridades de saúde devem aumentar o alcance e nível das atividades de pesquisa para o uso das tecnologias da informação e comunicação em saúde, através do estabelecimento de agendas de pesquisa. 
No âmbito internacional, existe a necessidade do estabelecimento de um recurso de avaliação que deveria estar sob os auspícios de organizações como Wonca ou OMS.

\section{Impacto sobre os Serviços de Saúde Rural - Acesso aos Serviços e a Prestaçáo de Serviços}

16. Os serviços de tecnologia da informaçáo e comunicaçáo devem ser usados para apoiar e melhorar, mas não para substituir a prestação dos serviços locais de cuidados de saúde para as comunidades rurais.

17. Os padróes de serviços de telessaúde devem, sempre que possível, refletir e apoiar os padróes de referência e acesso existentes nas comunidades rurais para os níveis secundário e terciário.

\section{Impacto sobre os Serviços de Saúde Rural - Recrutamento e Retençáo}

19. Os programas que abordam o recrutamento e retenção de profissionais de saúde para as comunidades rurais devem colocar uma alta prioridade nas tecnologias da informação e comunicação que podem melhorar o ambiente de trabalho e o estilo de vida dos trabalhadores de saúde e das comunidades rurais.

\section{Impacto sobre os Serviços de Saúde Rural - Educaçáo Continuada}

20. As tecnologias de informação e comunicaçáo instaladas para fornecer serviços de telessaúde devem ser disponibilizadas para os trabalhadores de saúde rurais para acesso à educação e formação contínua.

21. Os programas de telessaúde têm o potencial para desempenhar um papel crucial, fornecendo educação continuada como parte integrante do processo de consulta. Este deve ser estimulado pela:

- promoção de um elevado grau de colegialidade no processo de consulta,

- permitindo o aspecto educativo no orçamento e no processo de gestáo do tempo e

- incentivando ativamente a participação do trabalhador da saúde de referência no processo de consulta.

\section{Preâmbulo}

\section{Finalidade do documento}

Telessaúde é a prestação de serviços de saúde a distância - como tal, era o foco predominante da edição anterior deste documento de orientação política. Embora muitos dos temas e recomendaçôes dessa edição sejam também amplamente aplicáveis ao tema ampliado da tecnologia da informação e comunicação, o mundo mudou, em particular neste campo, tendo visto grandes mudanças em um curto período.

Esta declaração política tem como objetivo fornecer diretrizes que garantam, quando o planejamento é empreendido para os serviços de tecnologia da informação e comunicação rural, a gama de tecnologias da informação e comunicação consideradas apropriadas para as necessidades dos cuidados de saúde locais e serviços. Os contextos cultural e social em que os serviços serão introduzidos também devem ser levados em consideração.

Os membros da Wonca Rural Information Technology Exchange (WRITE) prepararam o documento. A WRITE foi formada na Primeira Conferência Internacional sobre Medicina Rural, em Xangai, em maio de 1996 por um grupo de médicos rurais com interesse no uso adequado da tecnologia da informação em saúde. 


\section{Compromisso Político e Financiamento}

O financiamento para os serviços de saúde rurais em uma base per capita é significativamente menor do que para os serviços de saúde urbanos em muitos países desenvolvidos e em desenvolvimento. Qualquer planejamento ou decisão de financiamento para introduzir tecnologias da informação e comunicação para áreas rurais e remotas não deve ter um efeito adverso sobre os níveis de financiamento existentes para essas áreas. A introdução de tecnologias da informação e comunicação não deve ser usada como justificativa para a redução dos níveis de financiamento para outros serviços. Isso só pode ser evitado pelos governos, com uma política clara de compromisso de melhorar o estado de saúde de suas populaçóes rurais. Os níveis apropriados de financiamento para a saúde rural deve apoiar este compromisso político.

A WRITE acredita que a tecnologia da informaçáo e comunicaçáo tem o potencial para melhorar a qualidade dos cuidados de saúde nas áreas rurais de todo o mundo, apoia a sua aplicação em que o financiamento específico é disponibilizado e quando os serviços de tecnologia da informação e comunicação se mostram apropriados para apoiar ou melhorar os serviços locais de saúde rural.

\section{Antecedentes}

Uma das questóes mais críticas na saúde rural em todo o mundo tem sido a falta de acesso das comunidades rurais ao mesmo nível de serviços de saúde desfrutados por comunidades urbanas. Esta falta de acesso foi criada por um certo número de fatores, incluindo a falta de profissionais de saúde preparados para trabalhar nessas áreas, a distância do local de serviços de saúde e a falta de recursos adequados. O problema é ainda mais grave para os trabalhadores de saúde que escolhem trabalhar em comunidades rurais e remotas, pela falta de acesso à informação para apoiar a prestação dos serviços de saúde, para avaliar as necessidades e a eficácia dos sistemas de distribuição de saúde, educação, formação e apoio contínuo aos seus papéis, incluindo o apoio dos pares adequados. O resultado final muitas vezes é a maior rotatividade de pessoal.

A convergência das tecnologias de informação e comunicação criou um ambiente técnico, em que existem desenvolvimentos rápidos em tecnologias interativas e melhorado o acesso a conjuntos mais ricos de informação. Estas tecnologias combinam a comunicação de alta velocidade, possibilitadas pela tecnologia digital, baseadas em sofisticados programas e aplicaçóes de computador. O interativo desenvolvimento de aplicaçóes têm altos níveis de funcionalidade, e cada vez mais preços acessíveis. No entanto, o acesso aos serviços de telecomunicaçóes confiáveis e adequados, especialmente à rede de telefonia, na maioria das áreas rurais mundiais, é limitado e precisa ser atualizado. O primeiro passo é o de proporcionar serviços de telecomunicaçôes que permitirão a comunicação de voz, fax e de dados adequados para acesso à Internet. O próximo passo é a modernização dos serviços de telecomunicaçóes com os padróes digitais, e, em terceiro lugar, a longo prazo, disponibilizar serviços de banda larga, tornando-os acessíveis. É reconhecido que, para muitas comunidades rurais, não será econômico fornecer serviços além da telefonia básica. O impacto desses desenvolvimentos permanece incerto, e os custos podem ser ainda muito grandes para muitos países em desenvolvimento. No entanto, o potencial para estes desenvolvimentos tecnológicos, para permitir a entrega de informação eficaz dos custos e serviços de tecnologia da comunicaçáo, deve ser reconhecido em qualquer processo de planejamento e implementação para a introdução de tecnologias da informação e comunicação para as áreas rurais.

É importante implementar um nível de tecnologia que seja apropriado para curto prazo e metas de longo prazo de uma regiāo. Ao escolher o nível de tecnologia para uma determinada comunidade rural, em termos de utilidade e retorno sobre o investimento, há uma hierarquia de especificaçóes de métodos e necessidades de telecomunicaçôes para os planejadores e projetistas de sistemas a considerar. Estes são:

1. Voz - telefonia ponto a ponto

2. Voz - teleconferência multiponto

3. Troca de dados analógico - fax

4. Troca de dados digital - incluindo os arquivos, imagens, fluxos de dados

5. Em tempo real de alta banda larga, troca de dados multiponto 
A oportunidade existe agora para tecnologias da informação e de telecomunicaçóes para ter um impacto significativo na saúde das comunidades rurais. Em particular, a aplicação destas tecnologias para a saúde oferece o potencial para reduzir as barreiras da distância, custo, má distribuição dos serviços e falta de apoio para os trabalhadores de saúde que, atualmente, restringem o acesso de comunidades rurais para o nível dos serviços de saúde apreciados pela maioria das comunidades urbanas. No entanto, embora a tecnologia da informaçáo e comunicaçáo tenha sido promovida como um meio que tem o potencial de entregar ou melhorar a prestação dos serviços de saúde nas áreas rurais, não houve muitas evidências até o momento para apoiar esta afirmação. Em particular, há pouca evidência para sustentar o custo-benefício da introdução da tecnologia da informação e comunicação em áreas rurais. Essa escassez de evidências sugere que qualquer financiador, planejando apresentar serviços de tecnologia da informação e comunicação para as áreas rurais, deve avaliar cuidadosamente o impacto da tecnologia da informação e comunicação sobre o financiamento para ambos os serviços existentes e quaisquer serviços futuros.

Questóes específicas que precisam ser abordadas incluem:

- a falta de conscientização e treinamento no uso e aplicação da respectiva tecnologia da informação e comunicação por muitos trabalhadores de saúde rurais;

- projetos de investigação e avaliação precisam ser feitos para determinar o potencial das tecnologias da informação e comunicação emergentes;

- as questôes legais e regulatórias que podem agir como barreiras para a efetiva implementação de tecnologias da informação e comunicação precisam ser resolvidas;

- a presente infraestrutura de telecomunicaçôes e serviços para a maioria das áreas rurais não são confiáveis e são inadequados para a aplicação sistemática e eficaz para os serviços de saúde;

- o desenvolvimento de políticas de tecnologias da informaçáo e comunicação e iniciativas para a saúde são muitas vezes escalonados e fragmentados e poderiam beneficiar as alianças estratégicas entre os setores de saúde, com outro governo, educação e setores privados em escala local e mundial.

Pesquisas com trabalhadores de saúde rurais sugerem que esse grupo foi o primeiro a adotar essa tecnologia. Essa atitude será um fator importante que irá influenciar a introdução de serviços de tecnologia da informação e comunicação em comunidades rurais.

\section{Parcerias com a Comunidade e Avaliação das Necessidades}

É essencial que as necessidades e questôes locais sejam levadas em consideração na aplicação dos sistemas de tecnologia da informação e comunicação. Os fatores locais são fundamentais na determinação do que deve ser implementado. Sem este apoio local, a imposição de qualquer serviço de tecnologia da informação e comunicação é muito provável que seja vencida. A comunidade e os profissionais de saúde rurais são as fontes mais confiáveis de informaçóes sobre esses fatores. Os planejadores centrais não devem ignorar as opinióes e necessidades dos profissionais de saúde locais no planejamento e implementação dos serviços de tecnologia da informação e comunicação. Para fazer isso, deve-se arriscar na criação de um ambiente de serviço de saúde local que pode tornar-se severamente diminuído em sua eficácia em comparação com os serviços de nível anterior.

Para assegurar a implementação bem sucedida da tecnologia da informação e comunicação, os seguintes critérios devem ser atendidos:

- Antes da concepção e implementação de rede e infraestrutura de tecnologias da informação e comunicação, é essencial realizar uma avaliaçáo das necessidades para e com as comunidades envolvidas, com oportunidades adequadas para a entrada da comunidade rural e seus trabalhadores de saúde. Na avaliaçáo de necessidades, devemos olhar para as futuras necessidades da comunidade.

- A avaliação das necessidades deve ter uma visão holística da comunidade, além de uma avaliação global das necessidades de saúde da comunidade. Deve também levar em conta a situaçáo econômica e educacional da comunidade, e quaisquer outros fatores locais que terão impacto sobre a introdução de um sistema de tecnologia da informação e comunicação. 
- A avaliação das necessidades deve demonstrar claramente à comunidade que a introdução de tecnologias da informação e comunicação será adequada para a comunidade, tanto em termos de saúde, como em termos econômicos.

- A comunidade e seus profissionais de saúde devem ter indicado claramente que estão preparados para aceitar e apoiar a introdução de tecnologias da informação e comunicação.

Existe uma necessidade de todos os participantes terem alguma propriedade do processo e do produto em tecnologia da informação e comunicação. As comunidades rurais e seus trabalhadores de saúde devem ter o acesso adequado em todas as fases do desenvolvimento dos sistemas de tecnologia da informação e comunicação que lhes são destinados. Nessa abordagem de parceria, porque eles são mais afetados pelos sistemas de tecnologias da informação e comunicação que será instalado, na maioria dos casos, o equilíbrio de poder na tomada de decisóes sobre a tecnologia da informação e comunicação deve descansar no seio das comunidades rurais e não em qualquer agência central ou demais agências.

\section{Recomendação 1}

O planejamento dos programas de tecnologia da informação e comunicaçáo deve reconhecer e responder especificamente para as necessidades locais e expectativas da comunidade rural e dos trabalhadores da saúde. A participaçáo ativa das comunidades e dos trabalhadores rurais deve ser um componente integral em todas as fases do planejamento e implementaçáo do processo de tecnologias da informaçáo e comunicaçáo.

\section{Especificação e Design do Sistema}

A especificação e design de qualquer sistema de tecnologia da informação e comunicação para uma comunidade rural devem levar em conta fatores locais, tais como:

- o meio ambiente local - incluindo poeira, umidade e temperatura ambiente;

- infraestrutura física - incluindo serviços de telecomunicaçóes existentes (se houver), fonte de energia, condição e segurança dos edifícios dos serviços de saúde;

- posiçáo geográfica e isolamento - em especial a distância do centro de referência mais próximo e modalidades de transferência existentes e padróes de referência.

Todos têm um papel na determinação do que pode ser executado em um sistema de tecnologia da informação e comunicação.

$\mathrm{Na}$ primeira etapa de especificação e design do sistema de serviços de tecnologia da informação e comunicação para a saúde rural, é importante reconhecer que há retornos decrescentes à medida que aumenta o investimento em tecnologia da informação. Isso significa que, com baixo nível e custo relativamente baixo, as tecnologias têm o potencial para oferecer acesso a serviços de tecnologia da informação e comunicaçáo úteis para muitas comunidades rurais, especialmente os serviços baseados na Internet. É importante trabalhar dentro do orçamento disponível para uma comunidade ou região; portanto, a entrada ativa, a partir da comunidade, é essencial na implementação de qualquer sistema.

Planejadores e designers de tecnologias da informação e comunicação devem reconhecer o impacto econômico dos serviços de tecnologia da informação e comunicação na comunidade rural ao especificar componentes e critérios de desempenho para tecnologia da informação e comunicação. Muitas comunidades rurais não serão capazes de sustentar os serviços de tecnologia da informação e comunicação sem significativos subsídios do centro de financiadores. Isso não deve ser à custa de financiamento reduzido para outros serviços locais de saúde. Esta afirmação é consistente com o World Bank's May 1995 Operational Policy Directive on Telecommunications (Diretiva Operacional sobre Política de Telecomunicaçóes), que observa que

O Banco incentiva os governos a desenvolverem estratégias para estender os serviços de telecomunicaçôes para toda a população, incluindo os grupos menos privilegiados. Serviços que são considerados necessários para o desenvolvimento social ou por razóes de segurança, mas que não são rentáveis, até mesmo sob a entrada liberal e políticas de preços, podem ser fornecidos para grupos populacionais de baixa renda (incluindo rural), através de instalaçôes comuns ou tornado viável por meio limitado, com subsídios do governo. 
Os princípios do projeto devem assegurar o uso contínuo da tecnologia - um sistema que é muito complexo ou estranho cairá rapidamente em desuso. Projetistas de sistemas devem considerar que diferentes categorias de usuários podem necessitar ser acomodados em um sistema, e que um sistema não deve ser concebido apenas com os trabalhadores de saúde em mente como usuários finais.

Enquanto os fatores locais são muito importantes na concepção e especificação de um sistema de tecnologia da informação e comunicação, qualquer sistema projetado deve estar de acordo com normas e protocolos internacionalmente reconhecidos. Em vários níveis, a falta de dados e padrôes de protocolo de comunicação tem dificultado a introdução bem sucedida da tecnologia em vários campos da medicina. Se as comunidades estão fazendo melhor uso dos sistemas de tecnologia da informação e comunicação para melhorar os cuidados de saúde, é essencial que os sistemas consigam a integração máxima com os padrôes de comunicação regional, nacional e internacional e de dados estruturais de saúde. Este reconhece a necessidade de usar o sistema de tecnologia da informação e comunicação para, eficazmente, comunicar-se com áreas e comunidades para além do âmbito local das operaçóes.

\section{Recomendação 2}

Como parte integrante do estabelecimento de programas de tecnologia da informaçáo e comunicaçáo é o de assegurar que o sistema que é implementado adere às recomendaçóes de estrutura de dados e padróes de protocolos de comunicaçáo que proporcionam melhor conformidade com os sistemas regionais, nacionais e internacionais.

\section{Segurança e Confidencialidade}

Uma parte essencial de qualquer projeto de sistema de tecnologia da informação e comunicação é assegurar que toda a informação transmitida pelo sistema é absolutamente segura. Qualquer quebra de sigilo de informações de saúde sensíveis poderiam ser potencialmente desastrosas não só para os indivíduos, mas também para toda a comunidade. Designers de serviços de tecnologia da informaçáo e comunicação devem aderir às normas reconhecidas internacionalmente para a transferência segura de informaçôes de saúde, tais como HL 7 e Edifact. É importante que todos os protocolos de segurança possam ser implementados em nível local em uma comunidade rural, sem reduzir a integridade profissional e independência dos profissionais de saúde locais.

\section{Recomendação 3}

A especificaçáo de protocolos de segurança da tecnologia da informação e comunicação deve aderir aos padróes reconhecidos em nível internacional. A implementação destas normas náo deve impactar negativamente os trabalhadores de saúde rurais.

\section{Implementação}

\section{Questões locais}

O nível local de conhecimentos, habilidades e atitudes para a tecnologia da informação é um importante fator determinante na implementação de um sistema. Trabalhadores de saúde locais e planejadores devem estar cientes dos potenciais benefícios da tecnologia da informação e comunicação e as possibilidades de desenvolver serviços adequados dentro das limitações de recursos disponíveis. A formação dos trabalhadores de saúde locais, para alcançar um nível adequado de habilidades para 
operar e manter instalado os equipamentos de tecnologia da informaçáo e comunicaçáo deve ser visto como um componente imperativo de qualquer implementação de tecnologia da informação e comunicação.

A acessibilidade de recursos é inversamente proporcional à distância dos centros populacionais. Quando este princípio é aplicado à tecnologia da informação e comunicação, empresas de comunicação vão cobrar mais por acesso remoto a sistemas de telecomunicaçôes. Um problema de implementação chave é a responsabilidade de financiamento contínuo do serviço, em particular os custos recorrentes de comunicação, que não deve ser prejudicial para os atuais níveis de financiamento local para os serviços de saúde. Comunidades rurais e remotas não devem ser penalizadas com custos mais elevados para acesso a recursos de telecomunicaçôes. Tem sido uma prática econômica generalizada subsidiar as comunidades rurais e remotas em muitas áreas de infraestrutura de apoio, por exemplo, estradas, abastecimento de água. Os custos relativos de equalização do custo de fornecimento das telecomunicaçóes e de informação são muito menores e os benefícios potenciais são muito maiores.

No entanto, deve ser reconhecido que dentro da estrutura econômica atual da maior parte dos sistemas de cuidados de saúde, as maiores economias da implementação do telessaúde podem estar nos custos de viagem para pacientes em regiốes remotas. Quando as comunidades têm subsidiado a organizaçâo de viagens para a transferência de pacientes, essa economia pode ser facilmente identificada. Em sistemas de saúde que não pagam diretamente as despesas de viagem, que são de responsabilidade do paciente, será mais difícil medir qualquer economia direta.

Os custos mais elevados de implementação da tecnologia da informação e comunicação nas áreas rurais pode ser reduzida através da aplicação de métodos tecnológicos adequados como micro-ondas e transmissão de dados via satélite. Os referidos meios podem ainda ser demasiado caros para implementar em muitas regióes do mundo, mas são relativamente mais viáveis do que os métodos anteriores de comunicação. Existem agora vários exemplos de tais tecnologias "leap-frogging" nas regiōes em desenvolvimento, onde as infraestruturas baseadas em fio têm sido completamente ignoradas pela infraestrutura de rede sem fio.

\title{
Recomendação 4
}

Comunidades rurais devem ter acesso ao nível dos serviços de telecomunicaçóes e largura de banda capaz de fornecer pelo menos o nível mínimo de serviços de tecnologia da informação e comunicaçáo para as suas necessidades identificadas.

\section{Recomendação 5}

\author{
As comunidades rurais não devem ser penalizadas por quaisquer alteraçóes no ambiente econômico \\ local criadas quando os sistemas de comunicação são instalados para apoio às tecnologias da informaçáo \\ e comunicação.
}

\section{Compartilhamento de Recursos Local, Regional e Mundial}

A tecnologia da informação e comunicação oferece a possibilidade de construção de pontes para ligar as comunidades rurais, os trabalhadores da saúde e outros. O planejamento para a implementação também deve considerar o potencial para o compartilhamento de recursos entre o governo e os setores privados. Se o setor privado tem interesse em uma área rural (por exemplo, exploração mineral), então, muitas vezes, desenvolve infraestrutura de comunicação excelente para o pessoal de suporte. As comunidades locais devem considerar as relações que formam com o setor privado, nas quais essas oportunidades existem, e formar empreendimentos cooperativos em que ambos podem se beneficiar das melhores comunicaçôes. Ao fazer uso mais eficiente de recursos escassos durante a implementação de serviços de tecnologia da informação e comunicação, consideráveis reduçôes de custos podem ser realizadas por organizaçôes que compartilham o uso de base ampla de comunicaçôes. Estas mesmas questôes de redução de custos se aplicam para outras áreas além da prestação de cuidados de saúde. Administraçôes municipais, agências de aplicação da lei, administraçôes de serviços de 
saúde, administraçóes industriais ${ }^{*}$ podem todos os benefícios da infraestrutura aprimorada de comunicaçóes e reduzir os custos globais dos serviços de telecomunicações.

Questôes de implementação da parceria para os países em desenvolvimento têm uma perspectiva global. Todos os países devem ser encorajados a procurar relaçóes de parceria regional e mundial em tecnologia da informação e comunicação. Maria Oakes Smith, 1997 World Bank Learning e Leadership Center Fellow, Tecnologia da Informação e Ensino à Distância no World Bank Bulletin No 1, levanta a preocupação de alcance global. Este se refere a

país em desenvolvimento o acesso à prestação da saúde, educação, informação pública, serviços comerciais e outros que utilizam apropriada tecnologia de comunicação - e é de divulgação às partes mais pobres do desenvolvimento mundial que exige relaçôes mais fortes de parceria.

Também é importante não ignorar o potencial de parcerias regionais para os países em desenvolvimento. Parcerias regionais, sempre que possível, podem resultar em economias significativas no custo de infraestrutura de informação e comunicação. Oakes Smith enfatiza as questôes-chave da

parceria entre indústria, academia, fundaçóes, organizaçóes internacionais e com as partes interessadas dos países em desenvolvimento, necessárias para concretizar o potencial de aplicação bem sucedida de (telessaúde) nos países mais pobres e entre os pobres que precisam mais dos serviços de saúde.

Ao descrever o impacto da parceria, Oakes Smith prevê um

novo relacionamento de parceria baseado em um comparativo de rede, mas diferentes habilidades (que) apoiariam inovações na prestação dos serviços de saúde e espalhariam os riscos financeiros que precisam ser levados a perceber o potencial para aplicaçóes de telemedicina nas regióes mais pobres do globo.

Esta visão reconhece a realidade de que "a era da informação é interativa, instantânea e em rede, e acima de tudo mundial." $\mathrm{Na}$ tecnologia da informação e comunicação, a sustentabilidade dos programas regionais e mundiais dependerá da disposição dos participantes em compartilhar conhecimentos e conselhos livremente.

$\mathrm{O}$ acesso online a publicaçóes de textos médicos completos foi recentemente muito melhorado para as naçóes em desenvolvimento por iniciativa da OMS, em colaboração com um consórcio de editoras liderado pelo BMJ Publishing Group. Este recurso anteriormente proibitivamente caro foi disponibilizado a preços muito justos para algumas das naçóes mais pobres do mundo. Esta iniciativa é uma grande promessa para a melhoria na distribuição de informação médica essencial para as áreas mais necessitadas. Esperamos que este exemplo promova esforços filantrópicos semelhantes de colaboração em outros campos da tecnologia da informação e comunicaçáo, como com os principais editores de software de computador.

\section{Recomendação 6}

As comunidades rurais devem procurar trabalhar com outras partes interessadas em empreendimentos cooperativos para melhorar a infraestrutura das comunicaçóes locais.

\section{Recomendação 7}

Todos os países, em particular os países em desenvolvimento, devem ser encorajados a procurar ativamente relaçóes de parcerias regionais e mundiais com Universidades, empresas e fundaçóes em outros países para desenvolver e apoiar a disseminação de tecnologias da informaçáo e comunicação nas áreas rurais.

\footnotetext{
* Link Centre projeto entre a Canadian Association of Petroleum Producers (CAPP) e Shock Trauma Air Rescue Society (STARS), Calgary, Alberta, Junho de 1996.
} 


\section{Assuntos Regulatórios}

Os planejadores de serviços de tecnologia da informação e comunicação devem determinar a existência de quaisquer obstáculos regulamentares às tecnologias da informação e comunicação. Essas barreiras podem incluir:

- política de telecomunicaçóes que não suporta os subsídios para as áreas rurais;

- questóes de licenciamento interestadual ou internacional de trabalhadores da saúde, fornecendo serviços consultivo e de diagnóstico por serviços de telessaúde;

- a política que rege a responsabilidade legal para as decisóes de saúde pela saúde local e remota trabalhadores de cuidados com base em informações fornecidas por meio de serviços de telessaúde;

- a política de responsabilidade que regem legal para os trabalhadores de saúde interestadual ou internacional fornecendo consultoria e serviços de diagnóstico por serviços de telessaúde;

- política de Privacidade governar e segurança das informações de saúde que não faz reconhecer a utilização das telecomunicaçóes como uma modalidade de serviço de saúde entrega;

- partilha de regulamentos em matéria de informação médica que não reconhecem o uso das telecomunicaçóes como uma modalidade de prestação de serviços de saúde;

- regulamentos e normas para gestão da informação, incluindo codificação e sistemas de classificação, que não levam variaçóes rurais, culturais e étnicas em conta;

- padróes de tecnologia;

- políticas regionais/distritais sobre as modalidades de financiamento para obter informaçôes e tecnologia das comunicaçôes;

- políticas aduaneiras especiais de consumo e exportação que impedem a divulgação de tecnologias da informação e comunicação que seriam um benefício para a saúde das comunidades rurais.

É importante que todas as decisôes sobre o fornecimento de serviços de tecnologia da informação e comunicação para as comunidades rurais feitas com base na política existente ou na política destinada a superar essas barreiras reguladoras não tenham um impacto adverso sobre os serviços de saúde locais.

\section{Recomendação 8}

As questóes políticas e decisóes relativas à tecnologia da informação e comunicação não devem afetar adversamente a entrega local dos cuidados de saúde nas comunidades rurais.

\section{Recomendação 9}

As questóes de regulamentaçáo e barreiras que possam ter impacto sobre a entrega interestadual ou internacional dos serviços de tecnologia da informaçáo e comunicaçáo devem ser claramente identificadas e tratadas em um nível nacional.

\section{Reembolso}

Em muitos países, a taxa pelo serviço é o método predominante para o reembolso dos praticantes da saúde. Nestes países, os regulamentos dos órgãos nacionais normalmente proíbem o reembolso nos casos em que não houve a presença física ou o contato entre o médico e o paciente. Este princípio foi projetado para prevenir o abuso de faturamento, por exemplo, para aconselhamento por telefone. 
Nos países em que existe um sistema de saúde predominantemente financiado pelo Estado, o reembolso de serviços de saúde é frequentemente, em escala nacional, dividido regularmente com o fornecedor-comprador o nível de pagamento. Onde este sistema de financiamento da saúde está em vigor, acordos de financiamento contratuais existentes não podem dirigir a prestação de serviços por telessaúde. Mudanças regulamentares ou legislativas podem ser necessárias para permitir isso. Os administradores dos sistemas de gestáo e cuidados da saúde também precisam incorporar financiamento para os serviços de telessaúde em suas estruturas de pagamento.

O ganho máximo a partir do processo de teleconsulta é obtido quando o profissional de saúde referido está incluído no processo. Se o trabalhador de saúde referido é um praticante independente, então, deve considerar-se o fornecimento adequado do reembolso ao trabalhador referido. Exceçóes a este princípio, que são levantadas pelos órgáos de financiamento com base de que não há precedente, devem ter vigorosa oposição com a observação de que este é um modelo totalmente novo de consulta.

\section{Recomendação 10}

O planejamento e implementaçáo de serviços de telessaúde devem abordar a questáo do reembolso das partes envolvidas no processo de teleconsulta.

\section{Suporte e Manutenção em andamento}

Os sistemas de tecnologia de informação e comunicação irão precisar de manutenção, uma vez instalados, e irão cair rapidamente em desuso, a menos que haja suporte técnico adequado. Financiadores de serviços de tecnologia da informação e comunicação rurais devem alocar níveis suficientes de financiamento recorrente para que os trabalhadores rurais da saúde tenham acesso ao suporte técnico contínuo. Sempre que possível este acesso deve ter o custo de não mais do que uma chamada telefônica local para o trabalhador de saúde rural. O acesso para solicitar reparaçáo e manutenção de equipamentos é fundamental.

É também essencial alocar fundos recorrentes para manutenção contínua, capital de depreciação, reinvestimento e atualizaçôes nos equipamentos de tecnologia da informação e comunicação instalados em comunidades rurais para os serviços de tecnologia da informação e comunicação. Existe uma necessidade contínua para atualizar os sistemas de tecnologia da informação e comunicação para acompanhar o desenvolvimento e as tendências mundiais.

O apoio técnico e diagnóstico remoto é cada vez mais empregado na tecnologia da informação e comunicação. Isso ajudará a reduzir os custos de suporte, mas, centralmente na base, os grupos de apoio devem ter conhecimento dos fatores locais que afetam seus usuários remotos e comunidades.

O custo de manutenção e atualização não deve ser subestimado - o custo total de valores de propriedade para tecnologia da informação e comunicação em termos de três a cinco anos, quando dividido tipicamente, demonstram que $80 \%$ do custo total são despesas de manutenção e atualizações. Novamente, estes custos não devem ser suportados desproporcionalmente pelas comunidades rurais.

\section{Recomendação 11}

O financiamento para a tecnologia da informação e comunicaçáo deve incluir dotaçóes para fornecer níveis recorrentes adequados de despesas para o apoio técnico, manutençáo em curso e modernizaçáo de equipamentos. 


\section{Sensibilização e Formação}

Além de serem providos de suporte técnico, os trabalhadores de saúde rurais devem ser providos de adequada educação e formação, que lhes permitam operar instalações de tecnologia das informaçóes e comunicação. É importante que a atitude dos profissionais de saúde rurais seja alvo de educação e formação para criar expectativas realistas sobre o desempenho dos serviços de tecnologia da informação e comunicação.

Há uma falta geral de consciência por parte dos trabalhadores de saúde rurais em relação a tecnologias da informação e comunicação disponíveis e suas aplicações para a saúde. Estratégias de implementação de tecnologias da informação e comunicação devem identificar a necessidade de formação no:

- funcionamento das tecnologias de comunicação para suportar aplicaçôes de saúde;

- aplicação das tecnologias para a educaçáo continuada dos profissionais de saúde;

- aplicação das tecnologias aos serviços clínicos de saúde; e

- aplicação das tecnologias para avaliar e melhorar a prestação de serviços de saúde.

Cada uma dessas áreas tem questôes específicas que estáo associadas.

\section{Recomendação 12}

Uma promoçáo abrangente e programa de treinamento no uso e aplicaçáo de tecnologias da informaçáa e comunicaçáo para a saúde, incluindo a disseminaçáo de experiências de projetos existentes, sáo componentes obrigatórios de qualquer projeto de tecnologia da informaçáo e comunicaçáo. Tais programas devem reconhecer as necessidades diversas e variadas dos trabalhadores de saúde rurais e os clientes e as comunidades para as quais prestam serviços.

\section{Recomendação 13}

Todos os projetos que envolvam a aplicação de tecnologias da informaçáo e comunicação para a saúde, que são financiados por autoridades nacionais de saúde, devem ser obrigados a ter formaçáo na operaçáo da tecnologia integrada dentro do projeto e ser educados nas questóes culturais e organizacionais que são partes integrantes do sucesso na implementaçáo de tecnologia da informaçáo e comunicaçáo.

\section{Avaliação}

Há muito pouca evidência internacional que avalia a eficácia da atividade de tecnologia da informação e comunicação na prestaçấo de serviços primários de saúde, particularmente para as comunidades rurais em países em desenvolvimento. A evidência é necessária para justificar o gasto de recursos escassos em programas caros que não podem melhorar os serviços de saúde locais. Os critérios de avaliaçáo devem ser projetados para medir o processo, resultados e impacto da implementação e o desempenho dos serviços de tecnologia da informação e comunicação instalados nas comunidades rurais. Esses critérios também devem levar em conta a cultura e variaçóes étnicas nas comunidades rurais.

\section{Recomendação 14}

O desenvolvimento e mediçáo dos critérios de avaliaçáo do processo, impacto e resultado dos serviços de tecnologia da informaçáo e comunicaçáo devem ser componentes fundamentais de qualquer programa de tecnologia da informaçáo e comunicação e devem ser baseados em fatores que sáo relevantes e importantes para as comunidades rurais envolvidas. 
Indicadores para medir o desempenho do desenvolvimento e entrega de serviços de tecnologia da informaçáo e comunicação são necessários. A coleção de dados sobre serviços de tecnologia da informação e comunicação é fundamental para garantir que os serviços de tecnologia da informação e comunicação sejam eficazes nos critérios clínicos, econômicos e de desempenho. Pesquisa e desenvolvimento em dados e itens para medir os critérios de avaliação são essenciais.

\section{Recomendação 15}

As autoridades de saúde devem aumentar o alcance e nível das atividades de pesquisa para o uso das tecnologias da informação e comunicaçáo em saúde através do estabelecimento de agendas de pesquisa.

Em nível internacional, existe necessidade do estabelecimento de um recurso de avaliaçáo que deve estar sob os auspícios de organizaçóes como Wonca ou OMS.

\section{Impacto da Tecnologia da Informação e Comunicações nos Serviços de Saúde Rural}

Os problemas que se seguem devem também ser levados em consideração na concepção e implementação dos serviços de tecnologia da informação e comunicação para o desenvolvimento das comunidades rurais.

\section{Acesso aos Serviços e Prestação de Serviços}

A introdução de serviços de tecnologia da informação e comunicação não deve ter um impacto adverso sobre o acesso aos serviços de saúde por parte das comunidades rurais. Planejadores devem levar em consideraçáo os efeitos da tecnologia da informação e comunicação sobre os níveis de habilidade e perícia local em comunidades rurais e as relaçôes entre trabalhadores de saúde locais e suas comunidades. O planejamento para os serviços de tecnologia da informação e comunicação nunca devem substituir os serviços de cuidados da saúde local prestados por trabalhadores de saúde rurais para a sua comunidade no nível local.

No entanto, qualquer prestação de serviço baseada em tecnologias da informação e comunicação deve complementar a referência existente e os padrōes de acesso entre as comunidades rurais e os prestadores de cuidados de saúde secundário/ terciário. Se não, então a comunidade e os trabalhadores de saúde podem ignorar os serviços de tecnologia da informação e comunicação que teriam um impacto significativo sobre a viabilidade dos serviços de tecnologia da informação e comunicação. Qualquer variaçáo de referência existente e padróes de acesso para os serviços de saúde devem ser plenamente justificados e explicados à comunidade rural.

As tecnologias da informação e comunicação têm um grande potencial na avaliação das necessidades de saúde das comunidades rurais e na prestação dos serviços de saúde para lidar com essas necessidades. No entanto, os critérios de avaliação e metodologias utilizadas devem levar em conta as variaçôes culturais locais e étnicas das comunidades rurais. As comunidades rurais devem estar envolvidas no estabelecimento de tais critérios. As alterações que são implementadas, como resultado de tais avaliaçóes, devem ser com o acordo das comunidades envolvidas.

\section{Recomendação 16}

Os serviços de tecnologia da informação e comunicaçáo devem ser utilizados para apoiar e melhorar, mas não para substituir a prestaçáo de serviços de saúde local para as comunidades rurais. 


\section{Recomendação 17}

Os padróes de serviços de telessaúde devem, sempre que possível, refletir e apoiar os padróes existentes de referência e acesso das comunidades rurais para os serviços secundários e terciários.

\section{Recomendação 18}

A avaliaçáo dos serviços de saúde em comunidades rurais, utilizando a tecnologia da informaçáa e comunicaçáo, devem considerar as questóes culturais e étnicas locais e negociar essas questóes com as comunidades.

\section{Recrutamento e Retenção}

A introdução dos serviços de tecnologia da informação e comunicação, em associação com as tecnologias da informação e telecomunicaçóes, tem o potencial para melhorar o recrutamento e retençáo de profissionais de saúde rurais, reduzindo a sensação de isolamento profissional vivida por muitos destes trabalhadores. No entanto, este potencial ainda não foi claramente estabelecido, e precisa ser cuidadosamente avaliado e pesquisado.

\section{Recomendação 19}

Os programas que abordem o recrutamento e retençáo de trabalhadores de saúde para as comunidades rurais devem colocar uma alta prioridade em tecnologias da informaçáo e comunicaçáo que podem melhorar o ambiente de trabalho e o estilo de vida dos trabalhadores de saúde e das comunidades rurais.

\section{Educação Continuada}

A instalação das tecnologias da informação e da comunicação proporciona aos trabalhadores de saúde rurais a oportunidade de acessar uma ampla gama de serviços de educação continuada remotamente. As oportunidades de educação a distância serão limitadas pelo desempenho técnico dos sistemas instalados. No entanto, os sistemas devem ser disponibilizados aos trabalhadores de saúde rurais como parte de uma estratégia global para reduzir o isolamento profissional.

\section{Recomendação 20}

As tecnologias da informação e comunicação instaladas para fornecer serviços de telessaúde devem ser disponibilizadas para os trabalhadores de saúde rurais para acesso à educaçáo e formaçáo contínua.

Os trabalhadores de saúde rurais podem desenvolver a percepçáo de que o telessaúde simplesmente representa uma oportunidade para os especialistas estenderem sua influência. Uma crítica para o telessaúde é que ele tem potencial para incentivar a dependência da entrada do especialista e náo promover uma atitude para resoluçáo de problemas entre os trabalhadores de saúde rurais. O Documento da Wonca sobre Política de Formação para a Prática Rural sublinha a necessidade de evitar a criação de tal ambiente de desamparo aprendido.

Esta mudança de atitude também se estende para os pacientes. Experiências da entrada de especialistas em gestão de problemas complexos tendem a elevar as expectativas de que o mesmo grau de consulta deve estar disponível nos casos 
que estão bem dentro das capacidades do trabalhador de saúde rural. Isto tem o potencial para reduzir as habilidades e a motivação do trabalhador de saúde rural

As consultas em telessaúde não devem ser um fórum para mostrar as habilidades do especialista, com pouco benefício do processo de consulta para o médico assistente. Para garantir que haverá benefícios mútuos do processo de telessaúde para todas as partes envolvidas, é necessário assegurar na dinâmica de uma consulta de telessaúde:

Fomentar uma forte atmosfera colegial entre a comunidade assistida e o especialista em consultoria. O respeito mútuo para as habilidades de cada partido aumenta muito a aceitação da entrada na consulta e na aceitação das recomendaçóes que surgem a partir da consulta.

Assegurar que todas as partes envolvidas em uma consulta de telessaúde reconheçam o potencial para a educação no processo.

as partes referentes se beneficiam da experiência do médico de família consultor, com a probabilidade de ser capaz de lidar com casos semelhantes no futuro com menos necessidade de consulta;

os benefícios da consultoria especializada a partir do conhecimento local do médico de família assistente sobre o paciente, expectativas, condiçôes e costumes locais. O especialista também desenvolve um maior nível de familiaridade com as habilidades e incertezas do médico assistente e as capacidades e recursos locais.

\section{Recomendação 21}

\section{Os programas de telessaúde têm o potencial de desempenhar um papel crucial por proporcionar educaçáo continuada como parte integrante do processo de consulta. Isso deve ser estimulado por:}

- promover um alto grau de colegialidade no processo de consulta;

- permitir o aspecto educativo no orçamento e processo de gerenciamento de tempo; e

- incentivar ativamente a participaçáo do trabalhador da saúde assistente no processo de consulta.

\section{Conclusão}

Não há garantias de que a introdução de serviços de tecnologia da informação e comunicação será automaticamente um benefício para os trabalhadores de saúde rurais e para as comunidades a que servem. No entanto, é possível, com um planejamento cuidadoso, maximizar o potencial dos serviços de tecnologia da informação e comunicação bem sucedidos e sustentáveis para as comunidades rurais. Este documento fornece recomendaçóes genéricas, para o planejamento da política de tecnologia da informaçấo e comunicação, que podem orientar os trabalhadores de saúde e moradores de comunidades rurais ao redor do mundo a alcançar este resultado.

A chave para atingir esse resultado desejável é que os planejadores incluam as comunidades rurais e seus trabalhadores em um papel de parceria, desde a fase de planejamento mais precoce possível dos serviços. A imposição de serviços de tecnologia da informação e comunicação para as comunidades que não foram envolvidas no planejamento é muito pouco provável que tenha sucesso ou que seja sustentável. Neste papel de parceria, é importante tratar de questôes-chave relativas à formação, financiamento e apoio contínuo da tecnologia da informação e comunicação, a partir da perspectiva da comunidade. A responsabilidade recai sobre os promotores e planejadores dos serviços de tecnologia da informação e comunicação para assegurar que a introdução de tecnologias da informação e comunicação não terá quaisquer efeitos adversos sobre a prestação dos cuidados de saúde e o bem-estar econômico das comunidades rurais.

Caso um projeto de tecnologia da informação e comunicação seja implementado como parte de uma parceria local, nacional, regional ou internacional, é imperativo que o financiamento para a avaliação de tecnologias da informação e comunicação seja um componente integrante do projeto.

Finalmente, os serviços de tecnologia da informação e comunicação nunca devem ser destinados a substituir a prestação de serviços direto em nível local por profissionais de saúde devidamente treinados e apoiados. Pelo contrário, a tecnologia da informação e comunicação deve ser um complemento valioso para o suporte do padrão de atendimento que é entregue às pessoas que vivem nas comunidades rurais do mundo. 


\section{Glossário}

Analógico

Largura da banda

Tecnologia da comunicação

Digital

EDIFACT

E-mail

Fax ou fac-símile

Full duplex voice

Half duplex voice

HL7

Tecnologia da informação

Internet

World Wide Web

WHO (OMS)
Um sinal, tendo um valor representativo, em vez de uma unidade, o qual assume uma variação contínua de valores. Dado analógico é muitas vezes transmitido como ondas sonoras. Isto significa que podem ser transmitidos através de padrão de redes de telefone ou rádio.

A capacidade de largura da banda de transmissão de dados por uma tecnologia de comunicação. Quanto maior for a largura da banda mais rápida é a transferência de dados, a qual geralmente é medida em bits de dados por segundo. Uma maior largura de banda é quase sempre mais cara para instalar e usar.

Tecnologias usadas para transmissão de dados. Podem ser analógicas ou digitais. Existe uma vasta gama de tecnologias de comunicação disponível. Estas se dividem em dois grandes grupos, baseado em cabo (que vai por terra) ou sem fio [wireless]. Tecnologias sem fio [wireless] incluem rádio, micro-ondas e satélite. Tecnologias sem fio geralmente exigem menos infraestrutura, mas podem ser mais caras para uso do que as tecnologias baseadas em cabos.

Dados digitais são transmitidos em bits de dados digitais, geralmente como "1s" e "0s" ou código binário. Exige uma conexão digital, ou devem ser convertidos em dados analógicos por um modem. Conexões digitais são muito mais rápidas do que as redes analógicas, mas são atualmente mais caras para instalação e manutenção.

Um protocolo de segurança e um conjunto de padrões que estão sendo desenvolvidos e implementados, principalmente na Comunidade Europeia. Não necessariamente compatíveis com HL 7.

Mensagens de texto eletrônicas trocadas entre computadores. Este pode ocorrer em nível local, através de uma rede ou em nível mundial na Internet. Pode operar eficazmente em qualquer velocidade de conexão. E-mails estão sujeitos a normas e protocolos para garantir que possam ser trocados, independentemente do sistema de computador dos usuários finais. Mensagens de e-mail também podem transmitir arquivos anexados.

A transmissão de material impresso sobre o padrão de linhas telefônicas entre equipamentos dedicados a fax.

Conexão de voz entre duas partes, na qual ambas as partes podem transmitir a fala (incluindo conversar) em qualquer altura, por exemplo, a maioria dos telefones.

Conexão de voz entre duas partes, na qual apenas uma parte pode falar a qualquer momento. Comum em transmissões de rádio.

Um protocolo de segurança e um conjunto de padrões que estão sendo desenvolvidos e implementados, principalmente nos EUA. Não necessariamente compatível com EDIFACT. HL7 é um protocolo de segurança e um conjunto de especificações para o intercâmbio do padrão de dados de saúde, projetado para facilitar a transferência de dados de saúde residente em diferentes e díspares sistemas de computador em um estabelecimento de saúde. HL7 facilita a transferência de resultados de laboratório, dados da farmácia e de outras informações entre diferentes sistemas de computação.

Tecnologias baseadas na utilização de computadores e outros circuitos integrados para processar os dados e produzir informação.

A Internet é uma rede mundial de computadores que fornece a infraestrutura para e-mail, a WWW e a uma série de outras modalidades. No contexto do presente documento, o uso do termo "Internet" geralmente se refere apenas a e-mail. As regiões rurais de diversas partes do mundo não têm a infraestrutura de telecomunicações para fornecer as velocidades de acesso necessárias para a WWW.

A World Wide Web (WWW) é a interface gráfica para a Internet. Ela exige uma conexão relativamente alta de velocidade para a Internet. A WWW é regida por um conjunto de normas e protocolos para garantir que poderá ser acessada independentemente dos sistemas de computadores dos usuários finais.

World Health Organisation (Organização Mundial da Saúde).

\section{Tradução para a versão brasileira: Nilson Massakazu Ando e Ricardo César Garcia Amaral Filho}

Rio de Janeiro: SBMFC, 2012 